\title{
Diacronie
}

Studi di Storia Contemporanea

$N^{\circ} 35,3 \mid 2018$

Gli strumenti di Clio

\section{Il 1911 in Maremma}

Le celebrazioni del Cinquantenario e il dibattito sull'“unificazione mancata"

\section{Elisa Tizzoni}

\section{(2) OpenEdition \\ 1 Journals}

\section{Edizione digitale}

URL: http://journals.openedition.org/diacronie/8871

DOI: 10.4000/diacronie.8871

ISSN: 2038-0925

Editore

Association culturelle Diacronie

Notizia bibliografica digitale

Elisa Tizzoni, « II 1911 in Maremma », Diacronie [Online], № 35, 3 | 2018, documento 3, Messo online il 29 septembre 2018, consultato il 22 avril 2019. URL : http://journals.openedition.org/diacronie/8871 ; DOI : 10.4000/diacronie.8871 


\section{Diacronie}

Studi di Storia Contemporanea

\section{$35,3 / 2018$}

Gli strumenti di Clio: uomini, luoghi e teorie della storia dalla tradizione critica alla comunicazione digitale

\section{Il 1911 in Maremma: le celebrazioni del Cinquantenario e il dibattito sull"'unificazione mancata"}

Per citare questo articolo:

TIZZONI, Elisa, «Il 1911 in Maremma: le celebrazioni del Cinquantenario e il dibattito sull'“unificazione mancata”», Diacronie. Studi di Storia Contemporanea : Gli strumenti di Clio: uomini, luoghi e teorie della storia dalla tradizione critica alla comunicazione digitale, 35, 3/2018, 29/09/2018,

URL: < http://www.studistorici.com/2018/09/29/tizzoni_numero_35/ >

Diacronie Studi di Storia Contemporanea $\rightarrow$ http://www.diacronie.it

Rivista storica online. Uscita trimestrale.

redazione.diacronie@hotmail.it

Comitato di direzione: Naor Ben-Yehoyada - João Fábio Bertonha - Christopher Denis-Delacour - Maximiliano Fuentes Codera Anders Granås Kjøstvedt - John Paul Newman - Deborah Paci - Niccolò Pianciola - Spyridon Ploumidis - Wilko Graf Von Hardenberg Comitato di redazione: Jacopo Bassi - Luca Bufarale - Gianluca Canè - Fausto Pietrancosta - Alessandro Salvador - Matteo Tomasoni Diritti: gli articoli di Diacronie. Studi di Storia Contemporanea sono pubblicati sotto licenza Creative Commons 3.0. Possono essere

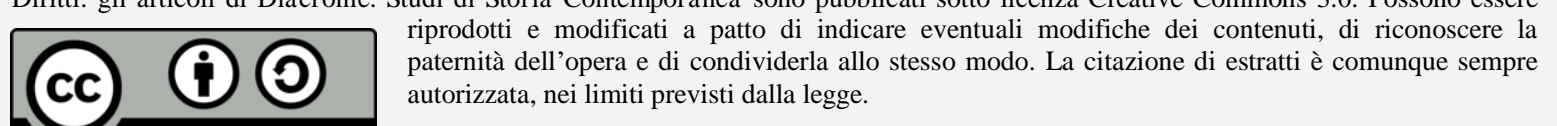




\title{
3/ Il 1911 in Maremma: le celebrazioni del Cinquantenario e il dibattito sull'"unificazione mancata"
}

\author{
Elisa TIZZONI
}

Prendendo spunto dalle recenti acquisizioni della nuova storiografia del Risorgimento, questo contributo esamina $\mathrm{i}$ contenuti e le modalità di svolgimento delle celebrazioni del cinquantesimo anniversario dell 'Unificazione italiana nella Maremma grossetana. Le peculiari condizioni politiche e socio-economiche di quest'area offrono l'occasione per sviluppare una riflessione sulla pluralità di significati associati alla ricorrenza e sul carattere contraddittorio delle manifestazioni giubilari, che esaltarono 1'Italia Giolittiana e nello stesso tempo evidenziarono la "mancata unificazione" delle aree periferiche meno sviluppate

\section{Introduzione}

A partire dagli anni Novanta storici di diversa formazione hanno alimentato un filone di studi che si situa a cavallo tra storia politica, culturale e sociale, e che ha come oggetto le festività civili e le commemorazioni in quanto strumenti di "nazionalizzazione delle masse" e legittimazione delle classi dirigenti nel contesto dello stato-nazione europeo tra XIX e XX secolo¹.

In questo ambito di ricerca, l'Italia liberale offre uno dei più frequentati terreni di studio, beneficiando dell'introduzione di nuovi quadri interpretativi definiti dalla "nuova storiografia" del Risorgimento ${ }^{2}$ e del rinnovato interesse per il processo di unificazione e consolidamento del Regno

\footnotetext{
${ }^{1}$ BAIONI, Massimo, «Identità nazionale e miti del Risorgimento nell'Italia liberale. Problemi e direzioni di ricerca», in Storia e problemi contemporanei, 22, 11/1998, pp. 17-40; PAGGI, Leonardo (a cura di), Le memorie della Repubblica, Firenze, La Nuova Italia, 1999; RIDOLFI, Maurizio, «Feste civili e religioni politiche nel 'laboratorio' della nazione italiana (1860-1895)», in Memoria e Ricerca, 5/1995, pp. 83-108; ID., Le feste nazionali, Bologna, Il Mulino, 2003; IRACE, Erminia, Itale glorie, Bologna, Il Mulino, 2003; BAIONI, Massimo, CONTI, Fulvio, RIDOLFI, Maurizio (a cura di), Celebrare la nazione. Grandi anniversari e memorie pubbliche nella società contemporanea, Milano, Silvana Editoriale, 2012; SOPER, Steven C., Building a civil society: associations, public life, and the origins of modern Italy, Toronto, University of Toronto Press, 2013.

${ }^{2}$ All'interno della vastissima produzione sull'argomento si rimanda ad alcune agili sintesi: ALBERGONI, Gianluca, «Sulla "Nuova Storia" del Risorgimento: Note per una discussione», in Società e Storia, 120, 2008, pp. 349-366; BANTI, Alberto Mario, GINSBORG, Paul, Per una nuova storia del Risorgimento, in IID. (a cura di), Storia d'Italia, Annali 22: Il Risorgimento, Torino, Einaudi, 2007, pp. XXIII-XLI; KÖRNER, Axel, RIALL, Lucy, «Introduction: the new history of Risorgimento nationalism», in Nations and nationalism, 15, 3/2009, pp. 396401; sulla dimensione transnazionale applicata con esiti particolarmente fruttuosi allo studio del Risorgimento italiano si veda: JANZ, Oliver, RIALL, Lucy, «Introduction», in Modern Italy, special issue The Italian Risorgimento: transnational perspectives, 19, 1/2014, pp. 1-4; sulla rielaborazione del "mito
} 
d'Italia seguito al centocinquantenario dalla sua fondazione3. Una prima stagione di studi ha condotto una intensa opera di de-costruzione 4 nei confronti della memoria pubblica del Risorgimento consolidatasi in epoca liberale attraverso l'accostamento di riferimenti simbolici e temi propri di culture politiche diverse, se non confliggenti, e finalizzata ad affermare un patriottismo congeniale alle esigenze delle élites e della dinastia sabauda, secondo una visione sostanzialmente negativa della "spettacolarizzazione della politica" che è stata probabilmente influenzata dalle teorizzazioni di Jürgen Habermas5.

Di contro, i più recenti studi sui contenuti del "mito risorgimentale" e le modalità della sua commemorazione hanno esaltato la dimensione plurale delle "narrazioni" diffuse nelle innumerevoli "piccole patrie"6 dell'Italia liberale7.

In primo luogo, è stato notato che le forze politiche che, per ragioni storiche e ideologiche, non si riconoscevano nelle forme istituzionali del Regno d'Italia (repubblicani e socialisti in primis), introdussero un proprio calendario di ricorrenze parallelo rispetto a quello sancito dalle autorità nazionali e, nel contempo, reinterpretarono le festività ufficiali alla luce del proprio patrimonio valoriale e delle specificità locali, forti di una esperienza amministrativa nelle istituzioni municipali che ne aveva ridimensionato il carattere antisistema ${ }^{8}$.

Pertanto, le declinazioni della memoria pubblica del Risorgimento nelle periferie offrono promettenti spunti di indagine, evidenziando, in particolare come l'establishment locale abbia esercitato una «funzione di mediazione tra gli interessi locali, dei quali si sentivano direttamente responsabili, e la politica nazionale, alla quale intendevano partecipare», che, nel caso delle

risorgimentale" a partire dall'epoca liberale sino all'età repubblicana: BAIONI, Massimo, Risorgimento conteso. Memorie e usi pubblici nell'Italia contemporanea, Reggio Emilia, Diabasis, 2009.

${ }^{3}$ BRACCO, Barbara, CASALENA, Maria Pia, a cura di, L'Italia in posa. Il $150^{\circ}$ e i problemi dell'unità nazionale tra storiografia e rappresentazione sociale, Bologna, Archetipo libri-Gedit edizioni, 2011; BAIONI, Massimo, "Anniversaries and the Public Uses of the Risorgimento in Twentieth-Century Italy», in Journal of Modern European History, 9, 3/2011, pp. 397-414.

${ }^{4}$ Il metodo de-costruttivista, partendo dalla trasposizione in ambito storiografico del concetto di déconstruction utilizzato dal filosofo francese Derrida per definire il processo con il quale sono messe in luce le inevitabili omissioni e le intime incoerenze, per lo più inconsce, presenti nel testo scritto, è stato inizialmente impiegato dalla storia culturale e, più recentemente, da altri filoni (post-colonial studies, gender history etc.) allo scopo di identificare ed interpretare come fatti storici le contraddizioni, le rielaborazioni, le soggettivizzazioni presenti nelle fonti scritte $\mathrm{e}$, più in generale, in quelle fonti maggiormente soggette a processi di elaborazione e rielaborazione. TOPOLSKI, Jerzy, Metodologia della ricerca storica, Bologna, Il Mulino, 1975; CAPLAN, Jane, «Postmodernism, Poststructuralism, and Deconstruction: Notes for Historians», in Central European History, 22, 3-4/1989, pp. 260-278; EDWARDS, Carole, «Réalité ou fiction? L'histoire à l'épreuve du postmodernisme», in European Review of History: Revue européenne d'histoire, 18, 4/2011, pp. 487-498; FAZZI; Patrizia, «Narrare la storia: la lezione di Jerzy Topolski», in Diacronie. Studi di Storia Contemporanea, 22, 2/2015, URL: < http:// diacronie.revues.org/2082 > [consultato il 25 luglio 2018].

${ }^{5}$ HABERMAS, Jürgen, L'Espace public. Archéologie de la publicité comme dimension constitutive de la société bourgeoise, Paris, Payot, 1997 [ed. orig.: 1962], p. 3.

${ }^{6}$ CAVAZZA, Stefano, Piccole patrie. Feste popolari tra regione e nazione durante il fascismo, Bologna, Il Mulino, 1997.

${ }^{7}$ VITTONATTO, Giorgia, Il Capanno di Garibaldi: culto del Risorgimento, memoria locale e cultura politica a Ravenna, Ravenna, Longo, 2005; FIORINO, Vinzia, RENZONI, Stefano, La Patria in marmo. I monumenti nazionali a Pisa, Pisa, ETS, 2006; MORANDI, Matteo, Garibaldi, Virgilio e il violino. La costruzione dell'identità locale a Cremona e Mantova dall'Unità al primo Novecento, Milano, FrancoAngeli, 2009; MANCUSO, Claudio, Ritualità pubblica e religioni civili in Sicilia (1860-1911), Palermo, Edizioni La Zisa, 2013.

${ }^{8}$ GORI, Annarita, The Risorgimento in Contemporary Italy, History, politics and memory during the national jubilees (1911-1961-2011), in MAMMONE, Andrea, PARINI, Ercole Giap, VELTRI, Giuseppe (eds.), The Routledge Handbook of Contemporary Italy, London - New York, Routledge, 2015, pp. 305-315. 
commemorazioni, si esplicava mediante l'innesto di temi squisitamente locali all'interno della narrativa risorgimentale imposta dall'alto9.

Nel contesto di studi sinteticamente descritto, le diverse prospettive di indagine applicate allo studio delle festività laiche nell'Italia postunitaria convergono nell'assegnare un ruolo paradigmatico al giubileo del 1911, «culmine dell'esaltazione dell'Italia liberale» ${ }^{10}$.

Il cinquantesimo anniversario dell'unificazione, difatti, venne a cadere in un anno, il «fatale 1911», nel quale ricorrono eventi con un alto valore periodizzante per la storia d'Italia ${ }^{11}$.

La guerra di Libia, in particolare, accelerò il processo di definizione dei diversi patriottismi in gestazione nei decenni precedenti, ricorrendo ad un ampio ventaglio di riferimenti ideali che certamente non si esauriscono nella vulgata nazionalista giunta alla ribalta con la ripresa dell'attività coloniale $^{12}$.

Il significato politico e culturale delle celebrazioni del cinquantenario, inoltre, sarebbe caratterizzato da una duplice prospettiva, rivolta sia al passato recente che al futuro del Regno d'Italia, dal momento che la consacrazione definitiva del "mito nazionale" fu accompagnata dall'esigenza di tracciare un bilancio riguardo il percorso di modernizzazione affrontato dal giovane stato ${ }^{13}$.

Riguardo il primo aspetto, è interessante rilevare che le celebrazioni promosse dalle autorità centrali riproposero forme di sincretismo tra le diverse esperienze risorgimentali elaborate nei decenni precedenti, nelle quali, grazie all'apporto dei maggiori intellettuali dell'epoca, le differenze ideologiche erano poste in secondo piano dal comune riferimento all'ideale della patria e si ammettevano nel pantheon degli eroi nazionali anche figure problematiche, come quelle di Garibaldi e Mazzini ${ }^{14}$.

Per quanto concerne la riflessione retrospettiva sui primi cinquant'anni del Regno d'Italia, secondo le fonti dell'epoca sia la maggioranza sia le opposizioni riconoscevano ai primi governi liberali il merito di aver compiuto progressi sulla via della modernizzazione, che furono documentati ed esaltati in occasione delle esposizioni organizzate nelle città che avevano svolto il ruolo di capitali del Regno (Torino, Firenze, Roma) ${ }^{15}$.

\footnotetext{
${ }^{9}$ ANGHELÉ, Federico, Educare alla patria: il discorso elettorale nell'Italia liberale, in ARISI ROTA, Arianna, FERRARI, Monica, MORANDI, Matteo (a cura di), Patrioti si diventa. Luoghi e linguaggi di pedagogia patriottica nell'Italia unita, Milano, Franco Angeli, 2009, pp. 131-142, p. 133.

${ }^{10}$ RIDOLFI, Maurizio, Almanacco della Repubblica. Storia d'Italia attraverso le tradizioni, le istituzioni e le simbologie repubblicane, Milano, Bruno Mondadori, 2003, p. 48.

${ }^{11}$ CARACCIOLO, Alberto, Il "fatale millenovecentoundici": Roma ed Europa tra mostre e congressi, in PIANTONI, Giovanni (a cura di), Roma 1911, Roma, De Luca, 1980, pp. 39-44.

${ }^{12}$ Ibidem.

${ }^{13}$ GENTILE, Emilio, La Grande Italia. Ascesa e declino del mito della nazione nel XX secolo, Milano, Mondadori, 1997.

${ }^{14}$ Esemplare, a questo riguardo, l'opera di Giovanni Pascoli, cantore ufficiale delle celebrazioni giubilari. LEVIS SULLAM, Simon, L'apostolo a brandelli. L'eredità di Mazzini tra Risorgimento e fascismo, Bari, Laterza, 2010.

${ }^{15} \mathrm{Ci}$ si riferisce all'Esposizione etnografica delle regioni e alla Rassegna internazionale d'arte contemporanea tenutesi a Roma; all'Esposizione internazionale delle industrie e del lavoro, che ebbe luogo a Torino offrendo una rappresentazione del tessuto produttivo italiano dell'epoca; alla Mostra del ritratto italiano dalla fine del XVI secolo al 1861 e all'Esposizione internazionale di floricoltura, ospitate a Firenze. PICONE, Mariantonietta, PETRUSA, Maria, PESSOLANO, Raffaela, BIANCO, Assunta, Le grandi esposizioni in Italia, 1861-1911: la competizione culturale con l'Europa e la ricerca dello stile nazionale, Napoli, Liguori, 1988; per approfondimenti si rimanda inoltre al catalogo ufficiale delle esposizioni: S.n., Le esposizioni del 1911 a Torino, Roma, Firenze, Milano, Treves, 1911.
} 
Nello stesso tempo, non mancò, soprattutto da parte dei socialisti, la denuncia dei perduranti ritardi di sviluppo che affliggevano numerose regioni d'Italia e che stridevano drammaticamente con la retorica ottimista delle celebrazioni ${ }^{16}$.

Come ha notato Massimo Baioni,

Il giubileo della patria [...] fu in effetti il momento in cui tutti furono chiamati a fare un bilancio del percorso compiuto in cinquant'anni di vita unitaria. Sguardo nazionale e sguardo locale si intrecciavano inestricabilmente, mettendo in luce le dinamiche che si muovevano sotto l'apparente ripetitività di cerimoniali e linguaggi ${ }^{17}$.

Conseguentemente, nonostante la presenza di una regia complessiva per le manifestazioni giubilari a livello nazionale ${ }^{18}$, le manifestazioni commemorative rivelarono una complessità di significati sia al centro che in periferia tale da giustificare il riferimento a diversi "giubilei" nella letteratura sul tema ${ }^{19}$.

Come notato da Catherine Brice, risulterebbe semplicistico ricondurre il carattere frammentario delle manifestazioni giubilari alla debolezza del processo di costruzione statuale italiano nel quadro di una unificazione "incompiuta"; al contrario, non va sottovalutato il ruolo svolto dagli attori locali, decisivo per determinare l'orientamento e il successo delle iniziative adottate in periferia ${ }^{20}$.

Nel corso dei primi decenni postunitari, secondo Brice, grazie a riforme che stimolavano l'identificazione dei cittadini con le istituzioni municipali e degli eletti con la comunità di riferimento, in un'epoca nella quale l'appartenenza partitica costituiva un vincolo ancora piuttosto labile, la mitologia risorgimentale sarebbe stata efficacemente integrata nelle identità regionali e municipali sino a definire una «complementarietà tra piccole e grandi patrie» ${ }^{21}$.

Sulla scorta della letteratura esistente possiamo dunque affermare che lo sviluppo di una vera e propria "rete" per la commemorazione dei fatti risorgimentali capillarmente diffusa in tutta la Penisola, che coinvolgeva istituzioni, associazioni e singoli cittadini, fu solo in parte il prodotto dei disegni egemonici delle élites, configurandosi, piuttosto, come un "contenitore" per le

${ }^{16}$ GENTILE, Emilio, La Grande Italia, cit.

${ }^{17}$ BAIONI, Massimo, Rituali in provincia: commemorazioni e feste civili a Ravenna, 1861-1975, Ravenna, Longo Angelo, 2010, p. 99.

18 A questo proposito, Baioni sottolinea che prima del 1911 la gestione delle ricorrenze legate al Risorgimento era demandata pressoché esclusivamente alle istituzioni e agli attori locali. BAIONI, Massimo, «Mémoires publiques du Risorgimento dans l'Italie libérale. Un parcours historiographique», in Revue d'histoire du XIXe siècle, 44, 1/2012, pp. 151-161.

${ }^{19}$ GORI, Annarita, The Risorgimento in Contemporary Italy, cit.

${ }^{20}$ BRICE, Catherine, "Il 1911 in Italia. Convergenza di poteri, frazionamento di rappresentazioni», in Memoria e ricerca, 17, 34, 2/2010, pp. 47-62.

${ }^{21}$ Ibidem. Le riforme citate da Brice includono, ad esempio l'introduzione dell'elettività dei sindaci (nel 1888 per i capoluoghi di provincia e i comuni con popolazione superiore a 10.000 abitanti, estesa a tutti i comuni nel 1896) e la creazione di un embrionale sistema di welfare locale mediante la municipalizzazione dei servizi (legge 103 del 29 marzo del 1903). 
rivendicazioni e le speranze delle generazioni maggiormente toccate dalle trasformazioni dell'Italia postunitaria.

Muovendo da questi stimoli, la nostra ricerca si focalizzerà sulle celebrazioni del 1911 nella Maremma grossetana, un'area che aveva offerto un contributo notevole ai moti risorgimentali e che aveva riposto nell'unificazione la speranza di una possibile risoluzione di problemi economici e sociali di lunga durata.

Nel 1911, come vedremo, la "questione maremmana"22 sembrava aver assunto una improvvisa urgenza non solo per gli attori locali ma anche per le istituzioni centrali, mentre nuove tensioni scatenate da fattori di ordine economico e politico rendevano ancora più sofferta la partecipazione alla festa nazionale.

Le peculiari caratteristiche della Maremma grossetana offrono dunque interessanti spunti di riflessione, contribuendo a colmare le perduranti lacune nell'analisi dei significati delle manifestazioni giubilari in periferia ${ }^{23}$.

\section{La Maremma nel 1911: società, economia, ambiente}

L'analisi dei principali indicatori statistici raccolti in occasione dei censimenti attuati tra l'unificazione e il 1911 mostra come il territorio maremmano, a fronte di un complessivo incremento del numero di abitanti, sia stato interessato da un progressivo indebolimento di alcuni dei settori che costituivano i principali bacini di occupazione della popolazione residente ${ }^{24}$.

Un primo elemento di criticità era individuabile nel permanere di ampie aree paludose interessate dalla malaria e prive di infrastrutture e di servizi fondamentali, stante l'inadeguatezza degli interventi promossi dalle istituzioni d'epoca liberale, che alimentò sentimenti nostalgici nei confronti del "buon governo" dei Lorena ${ }^{25}$.

${ }^{22}$ Con tale espressione ci si riferisce al complesso dei ritardi e delle distorsioni nello sviluppo socioeconomico dell'area, nonché al relativo dibattito accesosi in sede istituzionale e negli ambienti intellettuali, in maniera analoga a quanto verificatosi per la "questione meridionale".

${ }^{23}$ I precedenti studi sulla dimensione locale delle celebrazioni del 1911 si sono concentrati prevalentemente sulle grandi città (cfr.: TEDESCO, Luca, «Roma 1911 e la disfida dei Cinquantenari», in Storicamente, 7, 35, 2011, URL: < https://storicamente.org/tedesco_roma_1911 > [consultato il 16 maggio 2018]; GORI, Annarita, Tra Patria e campanile. Ritualità civili e identità politiche a Firenze in età giolittiana, Milano, FrancoAngeli, 2014) e sul Meridione (RIZZO, Maria Marcella (a cura di), "L'Italia è". Mezzogiorno, Risorgimento e post-Risorgimento, Roma, Viella, 2013; MANCUSO, Claudio, La patria in festa. Ritualità pubblica civile in Sicilia (1860-1911), Palermo, La Zisa Edizioni, 2013), con l'importante eccezione offerta dalla ricerca condotta da Massimo Baioni su Ravenna, che offre interessanti analogie con il nostro caso di studio, come approfondiremo oltre (BAIONI, Massimo, Rituali in provincia, cit.).

${ }^{24}$ Per un esame puntuale degli indicatori statistici riguardanti il territorio della Maremma grossetana nel periodo in esame si rimanda ai fascicoli censuari riferiti al periodo 1861-1911.

${ }^{25}$ BARSANTI, Danilo, «Le bonifiche nell'Italia centrale in età moderna e contemporanea: profilo storico e prospettive di ricerca», in Rivista di Storia dell'agricoltura, 27, 2/1987, pp. 67-104; nello stesso numero della Rivista di Storia dell'agricoltura si veda anche: ROMBAI, Leonardo, «Orientamenti e realizzazioni della politica territoriale lorenese in Toscana. Un tentativo di sintesi», pp. 105-147. 
Le perduranti difficoltà ambientali ipotecarono fortemente lo sviluppo del settore agricolo, tradizionalmente caratterizzato da forme di agricoltura estensiva e scarsamente toccato dalla diffusione della mezzadria. Nella prima decade del nuovo secolo, in particolare, si registrò una diminuzione dei piccoli proprietari, probabilmente riconducibile all'emigrazione e al passaggio nelle fila dei salariati, il numero dei quali aumentò in maniera consistente, mentre, tra le rilevazioni censuarie del 1901 e 1911 acquistò notevole peso il bracciantato ${ }^{26}$.

Il commercio manteneva un ruolo ancora marginale, con produzioni legate a pastorizia e agricoltura ed una distribuzione basata sulla rete di piccoli e medi porti locali, che beneficiava solo parzialmente della presenza della ferrovia, ancora in fase di completamento ${ }^{27}$.

Tra la fine del XIX secolo e gli inizi del successivo, di contro, il settore dell'estrazione e lavorazione di minerali si consolidò, con effetti rilevanti sul tessuto sociale, il paesaggio e l'identità locali.

L'arrivo di flussi migratori provenienti dai vicini centri della Toscana e da altre aree d'Italia, attratti dalle possibilità di impiego presso gli impianti estrattivi e di lavorazione dei minerali, modificò la composizione sociale delle comunità maremmane e stimolò ulteriormente la crescita dei nuclei abitati e l'urbanizzazione della costa, già in corso nei decenni precedenti:

Improvvisamente, una società arcaica e ancora intessuta con mille legami comunitari di piccoli coltivatori precari, braccianti, pastori, lavoratori forestali si trovò nella necessità di dover convivere con una ben più complessa realtà territoriale, caratterizzata da una presenza capitalistica ed industriale avanzata, trapiantata su un fondale ancora pre-capitalistico e rurale ${ }^{28}$.

L'industria estrattiva maremmana, tuttavia, esposta agli sbalzi del mercato che si susseguirono tra Otto e Novecento con gravi effetti su salari, occupazione e sicurezza dei lavoratori, continuò ad essere minacciata dalla concorrenza di aree dotate di più efficaci infrastrutture e meglio collegate alle reti di trasporto.

L'acquisizione degli impianti da parte di gruppi stranieri scarsamente radicati sul territorio, inoltre, ostacolava la creazione di un vero e proprio indotto e, più in generale, limitava la crescita complessiva dell'economia locale ${ }^{29}$.

\footnotetext{
${ }^{26}$ BIANCIARDI, Luciano, CASSOLA, Carlo, I minatori della Maremma, Cernusco Lombardone, Hestia Edizioni, 1995; AMMINISTRAZIONE PROVINCIALE DI GROSSETO, Miniere e minatori. Il lavoro, la vita, le lotte nelle miniere della Maremma Grossetana dalla metà del secolo XIX al secondo dopoguerra, Milano, Electa Editrice, 1985; BOLDRINI, Fabrizio, Minatori di Maremma. Vita operaia, lotte sindacali e battaglie politiche a Ribolla e nelle colline metallifere (1860-1915), Roccastrada, Edizioni Effigi, 2006.

${ }^{27}$ ZAGLI, Andrea, Breve storia di Grosseto, Pisa, Pacini Editore, 2007;

${ }^{28}$ ROMBAI, Leonardo, CIUFFOLETTI, Zefiro, La Maremma tra '800 e '900. Una rivoluzione territoriale e sociale, in GUARDUCCI, Anna (a cura di), Orbetello e l'identità della Maremma '800-'900, Firenze, Centro editoriale toscano, 2003, pp. 9-26, p. 15.

${ }^{29}$ TOGNARINI, Ivan, Siderurgia e miniere in Maremma tra '500 e '900: archeologia industriale e storia del movimento
} 
Il 1911, nondimeno, fu contrassegnato da una pur momentanea espansione del comparto minerario dovuta a fattori internazionali, come l'incremento del prezzo della lignite, e a condizioni interne, legate all'acquisizione delle società operanti in zona da parte di potenti gruppi stranieri ${ }^{30}$.

La relativa posizione di forza acquisita dalle forze lavorative grazie al trend positivo nel settore minerario riacutizzò la conflittualità sindacale, con il protagonismo di gruppi repubblicani e socialisti locali; tuttavia, anche per il sostegno inadeguato da parte delle organizzazioni politiche maremmane, gli scioperi e le altre manifestazioni dei lavoratori, pur toccando momenti di forte drammaticità, nella maggior parte dei casi non ottennero risultati significativi ${ }^{31}$.

La geografia politica locale in quegli anni era caratterizzata dalla leadership dei Repubblicani, il partito storicamente più forte in Maremma, rappresentati in Parlamento da Pio Viazzi, eletto proprio nel 1911 nel collegio che per quattro legislature era stato assegnato a Ettore Socci, ex garibaldino prematuramente scomparso nel 1905. Come si approfondirà più avanti, la minoranza liberale-moderata lanciò una campagna stampa contro l'onorevole Viazzi accusandolo di aver tradito le aspettative dell'elettorato riguardo possibili interventi statali a favore della Maremma, sebbene Viazzi avesse effettivamente esposto le problematiche della sua terra d'origine in sede parlamentare e richiesto l'applicazione delle stesse provvidenze già concesse ad altre aree interessate da criticità ambientali e sociali analoghe.

Il partito socialista maremmano nel $1911 \mathrm{fu}$ a sua volta interessato da tensioni interne solo parzialmente riconducibili allo scontro tra anima riformista e massimalista e dovute in gran parte a contrapposizioni locali, accompagnate da un crescente attivismo dei gruppi anarchici della zona $^{32}$.

\section{Le celebrazioni del Cinquantenario nella Maremma grossetana}

Ricorrendo alla documentazione archivistica superstite ${ }^{33}$ e, soprattutto, allo spoglio della stampa locale ${ }^{34}$, ci soffermeremo sulle commemorazioni del cinquantenario dell'Unità e degli altri

operaio, Firenze, All'insegna del giglio, 1984; PREITE, Massimo, Le miniere in Maremma, in ID. (a cura di), Paesaggi industriali del Novecento: siderurgia e miniere nella Maremma toscana, Firenze, Polistampa, 2006, pp. 95108.

${ }^{30}$ Uno dei fatti più rilevanti fu il subentro della Societé Generale des Lignites en Italie alla Società delle Ferriere Italiane nella gestione delle miniere di Ribolla. BOLDRINI, Fabrizio, Minatori di Maremma, cit., pp. 43-44.

31 TURBANTI, Adolfo, «La classe operaia delle miniere maremmane. Professionalità, cottimi, rapporti sociali», in Italia Contemporanea, 188, 1992, pp. 435-455; LAURITO, Marco, SOLDATINI, Simonetta, Censimento delle fonti d'archivio per la storia del '900 nella provincia di Grosseto, Firenze, Società editrice fiorentina, 2004.

${ }^{32}$ Le critiche all'operato di Viazzi, congiuntamente con fattori di ordine nazionale (tra i quali l'ampliamento del suffragio, il dibattito sull'intervento militare in Libia etc.), nel 1913 furono responsabili del passaggio del collegio parlamentare al partito socialista. BOLDRINI, Fabrizio, Minatori di Maremma, cit., pp. 222 et seq.

${ }^{33}$ L'esame della documentazione archivistica prodotta dalle istituzioni locali nella Maremma grossetana è fortemente limitato dalle notevoli perdite che essa ha subito, in gran parte dovute alle distruzioni causate dal secondo conflitto mondiale, e da scelte conservative che talvolta limitano l'accesso e la consultazione. 
anniversari legati alla memoria del Risorgimento svoltesi durante il 1911 nella maremma grossetana, valutando in che termini le caratteristiche del contesto locale abbiano condizionato le iniziative attuate e plasmato la lettura delle vicende risorgimentali.

La partecipazione della Maremma grossetana alle celebrazioni giubilari avvenne infatti ad una duplice scala, quella nazionale e quella locale, come documentato da «L'Ombrone», che dedicò ampio spazio all'evento in virtù del suo orientamento filo-sabaudo.

Secondo il periodico, dunque, inviando propri rappresentanti o fornendo materiali illustrativi in occasione delle esposizioni e degli altri avvenimenti organizzati a Roma, Firenze, Torino, la comunità maremmana avrebbe potuto "riscattare" la propria reputazione di terra arretrata ed inospitale, corroborando, nello stesso tempo, le richieste di provvedimenti speciali a favore dell'area avanzate dalle istituzioni locali.

Si sottolineava, inoltre, l'entusiastica partecipazione della popolazione, memore del contributo offerto dai patrioti maremmani all'unificazione italiana:

Un grande plebiscito di esultanza, a cui fa solenne riscontro il voto plaudente di ogni paese civile, si eleva da tutte le parti del nostro paese [...] è giusto, è doveroso che anche dalla nostra Maremma, che come le altre regioni italiche con sublime slancio ed a costo dei più gravi sacrifici contribuì a questa nazionale unità, si levi una voce di esultanza per render più alto l'inno di Osanna alla Patria nostra ${ }^{35}$.

La partecipazione della Maremma alle iniziative giubilari di carattere nazionale ebbe una prima, concreta manifestazione nella presenza di un padiglione dedicato al grossetano presso l'Esposizione etnografica di Roma, all'interno del quale fu ricostruita la celebre tomba etrusca di Vetulonia $^{36}$ e furono esibiti «stemmi, emblemi ed attributi del mare, dell'agricoltura, dell'industria mineraria, che sono tra le fonti del lavoro e della ricchezza della provincia» ${ }^{37}$.

\footnotetext{
${ }^{34}$ I periodici di riferimento per l'area della Maremma grossetana nel periodo in esame sono rappresentati da "L'Ombrone», di orientamento liberal-moderato, «Etruria Nuova», organo dei repubblicani, e «Il Risveglio", settimanale socialista; questa ricerca si basa su un accurato spoglio dei primi due periodici citati, poiché gravi lacune documentarie hanno impedito uno spoglio sistematico de «Il Risveglio».

${ }^{35}$ R.O., «Le Feste Giubilari del Regno d'Italia», in L'Ombrone, 26 marzo 1911.

${ }^{36}$ Come vedremo anche nel successivo paragrafo, in quest'epoca il richiamo alla cultura etrusca assumeva un significato ambivalente nel processo di costruzione dell'identità nazionale e locale: da un lato, gli Etruschi erano raffigurati come la prima "stirpe nazionale" ad aver tentato di unificare la Penisola sotto un unico dominio; di contro, sull'onda della "etruscomania" diffusasi a partire dal tardo Settecento in conseguenza di clamorosi ritrovamenti e del crescente interesse per l'archeologia in Europa, si enfatizzavano i caratteri oscuri e misteriosi della loro civiltà, come avvenne effettivamente nel caso dell'immagine stereotipata della Maremma "maledetta" ancora presente nella pubblicistica dei primi del Novecento. HAACK, Marie-Laurence, «L'étruscologie au xxe siècle. Bilans historiographiques», in Anabases, 23, 2016, pp. 11-26; FORO, Philippe, L'Italie et l'Antiquité du siècle des Lumières à la chute du fascisme, Toulouse, Presses Universitaires Du Midi, 2017).

${ }^{37}$ S.a., «Il Padiglione Toscano all'Esposizione Etnografica di Roma», in L'Ombrone, 16 luglio 1911. La sezione dell'esposizione dedicata alle regioni d'Italia si articolava attorno a quadri che riproducevano i diversi paesaggi della Penisola, realizzati con la consulenza del noto pittore Galileo Chini, figura chiave del liberty italiano, mentre il noto architetto fiorentino Ugo Giusti collaborò alla progettazione del padiglione toscano.
} 
In occasione di quello che fu il momento più solenne delle celebrazioni giubilari romane, l'inaugurazione del Vittoriano, tenutasi il 4 giugno, giunsero nella capitale i sindaci e i rappresentanti di quasi tutti i comuni grossetani, con l'eccezione forzata dei municipi commissariati e con quella, più probabilmente politica ${ }^{38}$, di alcune località tra le quali Massa Marittima, dove il mazzinianesimo e il garibaldinismo avevano profonde radici e l'amministrazione municipale era saldamente governata dai repubblicani ${ }^{39}$.

La città di Grosseto, inoltre, aderì senza esitazioni alla "festa dei gonfaloni", una iniziativa promossa dal Touring club italiano che avrebbe dovuto tenersi nella capitale dal 18 al 20 settembre per ricordare la presa di Roma, predisponendo l'invio di un

rosso vessillo [...] dove il Grifo rampante ricorda il valore degli avi e la libera e vetusta origine sua [...] poiché fiammeggiante al libero sole di Roma intangibile, rievocherà anch'esso, fra l'esultanza delle città sorelle, pagine gloriose di eroismo scritte dalla patriottica Maremma nella storia del risorgimento italiano» ${ }^{40}$.

Per organizzare al meglio la partecipazione di Grosseto alla manifestazione fu istituito un comitato $^{41}$, il quale ricevette adesioni talmente numerose da richiedere la nomina di una commissione esecutiva, presieduta dal patriota Baldassarre Stragliati ${ }^{42}$, mentre la cittadinanza venne invitata a sostenere l'iniziativa con delle oblazioni, che sarebbero state solennemente ricordate da attestazioni raccolte in schede rilegate e depositate nell'Archivio Comunale ${ }^{43}$.

Restando nell'ambito delle manifestazioni organizzate a livello nazionale, ricordiamo infine che anche la Biblioteca Chelliana, su iniziativa della presidenza del Consiglio del Parlamento

S.a., «Grosseto e l'Esposizione del 1911», in L'Ombrone, 5 febbraio 1911; PASSERINI, Giuseppe L., La toscana alla Mostra delle regioni, Firenze, Guarneschi, 1911; BENZI, Fabio, MARGOZZI, Mariastella, Galileo Chini. Dipinti, decorazione, ceramica, teatro, illustrazione. Catalogo della mostra (Roma, 9 giugno-10 settembre 2006), Milano, Mondadori-Electa, 2006.

${ }^{38}$ Sebbene la cerimonia di inaugurazione fosse seguita da un corteo dall'intento"conciliatore", che rese omaggio alla statua di Garibaldi al Gianicolo e ai monumenti dedicati a Mazzini e ai caduti di Porta Pia, il Vittoriano fu percepito dalle forze democratico-radicali come un atto di ingiusta appropriazione della tradizione risorgimentale da parte di Casa Savoia. BRICE, Catherine, Le Vittoriano: Monumentalité publique et politique à Rome, Roma, École Française de Rome, 1998.

${ }_{39}$ S.a., «I Sindaci della nostra Provincia alla inaugurazione del monumento al Padre della Patria», in L'Ombrone, 11 giugno 2011.

${ }^{40}$ S.a., «La festa dei Gonfaloni», in L'Ombrone, 30 luglio 1911.

${ }^{41}$ Il Comitato, che ricevette numerose adesioni (comprese quelle del Sindaco della città Egidio Bruchi e di altri notabili locali), era guidato da una Commissione esecutiva della quale facevano parte, oltre a Stragliati, anche Corrado Andreini, Gino Bianchi, Giuseppe Del Fa, Virgilio Matteini, Martino Nencini, Luigi Ponticelli, Giuseppe Tombari, Leopoldo Tognetti, Gualtiero Ugazzi.

${ }^{42}$ Ibidem. Stragliati partecipò ai moti risorgimentali dapprima arruolandosi come volontario nel $10^{\circ}$ Reggimento nel 1859 e partecipando successivamente sia alla seconda spedizione Medici in Sicilia che alla terza Guerra d'indipendenza, come egli stesso ricorda in un libro di memorie intitolato Da Milazzo a Villaglori: Diari di Baldassare Stragliati, edito in Garibaldi e i Garibaldini, I, 1/1910, pp. 161-176.

${ }^{43}$ S.a., La festa dei Gonfaloni, in L'Ombrone, 6 agosto 1911; in realtà alla vigilia dell'evento il Touring club decise di annullare la festa dei gonfaloni, adducendo "motivazioni sanitarie", scatenando le proteste del Comitato costituito a Grosseto. S.a., "Cronaca di Grosseto», in L'Ombrone, 24 settembre 1911. 
Italiano, ricevette in dono i 15 volumi relativi alla pubblicazione Le assemblee del Risorgimento, edita nel 1911 nell'ambito delle iniziative culturali per la celebrazione del cinquantenario ${ }^{44}$.

Carattere regionale ebbe invece la commemorazione del Giubileo svoltasi a Firenze nel mese di maggio, alla presenza dei Reali e di numerosi notabili, che vide la partecipazione di sindaci e rappresentanti dei municipi toscani, incluso quello di Grosseto ${ }^{45}$.

Passando alle commemorazioni di carattere locale, le divisioni ideologiche ridimensionarono l'afflato unitario testimoniato da "L'Ombrone» in occasione delle celebrazioni di carattere nazionale, come dimostrato dall'astensione di repubblicani e socialisti dalle manifestazioni organizzate per la ricorrenza del 27 marzo, seppur con alcune eccezioni.

Ricorrendo nuovamente al periodico di orientamento monarchico, apprendiamo che ad Orbetello il Comune e la locale stazione postelegrafonica ricordarono il cinquantenario della patria attraverso la deposizione di corone presso i monumenti dedicati a Vittorio Emanuele II, Mazzini, Garibaldi ed altri eroi risorgimentali, ma l'omaggio reso a figure di riferimento per repubblicani e socialisti non impedì che entrambi i raggruppamenti si astenessero dal partecipare alla cerimonia ${ }^{46}$. Tuttavia, il consigliere socialista dell'amministrazione municipale di Orbetello responsabile delle questioni scolastiche impose la partecipazione alla cerimonia del 27 marzo a tutti gli alunni delle scuole elementari cittadine, minacciando di sospensione disciplinare gli eventuali assenti, riconoscendo dunque sul piano istituzionale l'importanza di una ricorrenza dalla quale, pure, si dissociava sul piano strettamente politico ${ }^{47}$.

Nel contempo, il carattere conflittuale della ricorrenza del 27 marzo, percepita come celebrazione della dinastia sabauda e dell'Italia plasmata dalle forze liberali monarchiche e conservatrici, trovò un riscontro indiretto nelle manifestazioni "sotto tono" organizzate a Massa Marittima, definita la "Brescia maremmana" per il precoce e profondo radicamento degli ideali garibaldini e mazziniani tra la popolazione ${ }^{48}$, dove l'anniversario dell'Unificazione fu ricordato solamente da un discorso pubblico tenuto dal preside delle scuole tecniche locali alle presenza di alunni e docenti e dall'esposizione della bandiera «da diversi uffici pubblici e da qualche privato» ${ }^{49}$.

\footnotetext{
${ }^{44}$ S.a., «Un cospicuo dono del Presidente della Camera alla Biblioteca Chelliana», in L'Ombrone, 30 luglio 1911.

${ }^{45}$ Grosseto fu rappresentata dall'avvocato Amilcare Concialini, presidente del locale Comizio agrario. S.a., «Le feste di Firenze e la rappresentanza del comune di Grosseto», in L'Ombrone, 14 maggio 1911.

${ }^{46} \mathrm{Nemo}$, «La vita in provincia. Cronaca orbetelliana», in L'Ombrone, 2 aprile 1911.

${ }^{47}$ Ibidem.

${ }^{48}$ BERTOLUCCI, Franco, Antonio Gamberi, ateo convinto e fermo socialista. Appunti per una biografia, in BERTOLUCCI, Franco, RONCO, Daniele (a cura di), Poesie per un "liberato mondo": antologia, Pisa, BFS, 2004, pp. 11-56; SOLDATINI, Simonetta, (a cura di), Risorgimento nazionale e patria locale. La raccolta documentaria di Gaetano Badii nell'Archivio storico comunale di Massa Marittima, Siena, Betti, 2008.

${ }^{49}$ Onorato, «La vita in provincia. Massa Marittima», in L'Ombrone, 2 aprile 1911.
} 
La ricorrenza assunse un significato maggiormente legato all'identità locale e estraneo alla contrapposizione tra monarchici e repubblicani presso Porto Santo Stefano, dove le cerimonie furono incentrate sulla presenza della nave ammiraglia Pisa presso il porto, emblema della città ${ }^{50}$.

Nel capoluogo ebbero luogo le manifestazioni più partecipate e spettacolari, come testimoniato nuovamente da «L'Ombrone», che descrive in maniera pittorica l'aspetto della città il 27 marzo:

Fino dalle prime ore del mattino la città era tutta imbandierata [...] Le vie presentavano un insolito aspetto di festività e di animazione". La festa si era protratta anche nella serata: "Nella serata la città era insolitamente illuminata. Decorazioni luminose a piccole lampade elettriche pendevano sulle facciate di molti edifici pubblici, lampioncini alla veneziana adornavano le finestre di molte case private. Il corso fu fino a tarda ora animatissimo ${ }^{51}$.

Nel capoluogo grossetano l'evento clou del programma di festeggiamenti era stato rappresentato dalla conferenza sul ruolo di Carlo Alberto nell'unificazione, tenuta presso l'Associazione monarchica dal notabile locale Achille Piccirilli ${ }^{52}$.

Le manifestazioni giubilari coinvolsero anche le frazioni del comune di Grosseto, ed in particolare il borgo collinare di Montepescali, dove la ricorrenza del 27 marzo fu celebrata con una sfilata della banda musicale cittadina e con un discorso pubblico tenuto da Giovanni Bavona, consigliere municipale residente nella piccola località ${ }^{53}$.

Per poter interpretare correttamente il significato assunto dalle celebrazioni del cinquantenario in Maremma e comprendere il legame tra memoria del Risorgimento, identità locale e nation building, può essere opportuno allargare lo sguardo alle altre ricorrenze risorgimentali ricordate nel corso del 1911, la prima delle quali in ordine di tempo fu l'anniversario della battaglia di Curatone e Montanara (29 maggio).

In Maremma, come in altre località della Penisola, le cerimonie sottolinearono il sacrificio dei caduti in battaglia, assurto a gesto di riscatto per l'intera nazione e per le comunità locali, l'adesione volontaria dei combattenti, la loro giovane età ${ }^{54}$.

Nel contempo, nel grossetano le commemorazioni posero particolare enfasi sulla provenienza toscana dei "martiri" di Curtatone e Monatanara quale ulteriore motivo di orgoglio da parte delle comunità locali, come avvenne anche nel capoluogo e in altre aree della regione ${ }^{55}$.

\footnotetext{
${ }^{50}$ Cieffe, «La vita in provincia. Porto S. Stefano», in L'Ombrone, 2 aprile 1911.

${ }^{51}$ S.a., «Il XXVII marzo», in L'Ombrone, 2 aprile 1911.

${ }^{52}$ Ibidem.

${ }^{53}$ S.a., «La vita in Provincia. Montepescali, 29 marzo», in L'Ombrone, 2 aprile 1911.

${ }^{54}$ CIPOLLA, Costantino, "Questi rubini campestri...". Lettura sociologica di una sconfitta vittoriosa, in CIPOLLA, Costantino, TAROZZI, Fiorenza (a cura di), Tanto infausta sì, ma pur tanto gloriosa. La battaglia di Curtatone e Montanara, Milano, FrancoAngeli, 2004, pp. 17-81; GORI, Annarita, "Le "Termopili toscane". La memoria di Curtatone e Montanara in età giolittiana», in Storia e Futuro, 30, 2012, pp. 3-27.

${ }^{55}$ BURZAGLI, Claudia, «Tra piccola e grande Patria. La costruzione della memoria di Curtatone e Montanara in Toscana», in Rassegna storica toscana, 52, 2/2006, pp. 267-300.
} 
Ricordando la partecipazione di un nutrito gruppo di volontari maremmani alla battaglia ${ }^{56}$, un proclama pubblico della Giunta comunale di Grosseto sottolineava efficacemente il legame tra identità cittadina e nazionale:

Carissimi!

Volgono per l'Italia giorni di grandi memorie e di legittimi entusiasmi attraverso patriottiche feste e manifestazioni solenni, con le quali, dalle più grandi città alle più umili borgate, si commemora nel modo più degno il cinquantenario dell'Unità sua con Roma capitale.

L'avvenimento, che è rievocazione della gloriosa storia del patrio riscatto, opera imperitura di uomini sapienti e generosi, che con tenacia di propositi e con eroici sacrifici, la vollero e la compirono, commuove più che mai al cader di date che ricordano dolorose e sventurate arditezze.

Il 29 maggio è appunto una delle date più memorande, e quanti sentono amor di patria ed apprezzano virtù di abnegazione, non possono non evocare la memoria dei prodi giovani toscani, che a Curtatone e Montanara, con indomito valore, pugnando uno contro cinque, caddero decimati senza abbandonare il posto.

Cittadini!

Onore ai caduti e nella gioia che giustamente ci allieta pel cinquantesimo anniversario di quella unità, che era l'ideale auspicato di questi eroi e che ci fu data dal senno e dal sangue dei nostri predecessori, vada loro fervido e perenne il nostro sincero tributo di riconoscenza e di affetti $i^{57}$.

Nel successivo anniversario della presa di Roma (20 settembre), Grosseto fu sede di eventi che assunsero un carattere particolarmente frivolo: mentre l'amministrazione municipale adempiva agli obblighi istituzionali inviando al sindaco di Roma un telegramma con il quale si aderiva ufficialmente alla celebrazione della ricorrenza, in città un comitato di cittadini decorò la via intitolata a Garibaldi e le strade limitrofe con festoni e luci e organizzò una corsa dei sacchi (la "corsa degli insaccati", una manifestazione tradizionale che si tiene ancora oggi nella cittadina), seguita da un concerto ${ }^{58}$.

Se a Grosseto la ricorrenza del 20 settembre fu ricordata coniugando folclore e solennità in maniera piuttosto originale, a Sorano le celebrazioni si svolsero rispettando pienamente i "canoni" fissati per le manifestazioni giubilari istituzionali: apertasi con un corteo di associazioni e cittadini direttosi vero l'abitazione del fratello di Domenico Egidi, caduto nella battaglia di

\footnotetext{
${ }^{56}$ NICCOLAI, Lucio, La Maremma nel Risorgimento: memoria del territorio, Arcidosso, Effigi, 2011.

${ }^{57}$ Archivio di Stato di Grosseto, Fondo Archivio Storico Post-Unitario del Comune di Grosseto, Serie 6 Carteggio Generale degli Affari, Faldone 21.

${ }^{58}$ S.a., «XX settembre», in L'Ombrone, 24 settembre 1911.
} 
Bezzecca, la giornata proseguì con una pubblica orazione, seguita dall'inaugurazione di una lapide in memoria di Garibaldi e da una lezione pubblica sulla storia del Risorgimento ${ }^{59}$.

Anche in questo caso le strade della cittadina furono addobbate a festa e percorse dalla fanfara, mentre un banchetto serale, conclusosi con fuochi d'artificio, rappresentò la componente più propriamente "ludica" della commemorazione.

La manifestazione svolta a Sorano offrì dunque un esempio paradigmatico delle celebrazioni risorgimentali, e giubilari in particolari, promosse dalla classe dirigente filo-governativa in periferia, non solo per le modalità con le quali si svolse ma anche e soprattutto per il fatto di inserire la figura di Garibaldi nel patrimonio identitario comune della nazione, riconducendo ad un'artificiosa unità la pluralità delle culture politiche che contribuirono all'unificazione italiana.

Le celebrazioni soranesi, inoltre, risentendo forse del clima politicamente teso che caratterizzava l'autunno del 1911, fornirono ai redattori de «L'Ombrone» l'occasione per criticare le strumentalizzazioni della memoria risorgimentale e gli estremismi del garibaldinismo radicale:

la commemorazione [...] ha dimostrato che qui ancora vibrano i sentimenti che provocarono la meravigliosa epopea del nostro risorgimento e vive la speranza e l'augurio che ogni senso di idealismo non sia soffocato dalle aspre competizioni affaristiche o opportunistiche che al presente determinano e regolano la vita e la politica60.

Riferendosi all'inaugurazione della lapide dedicata a Garibaldi, si aggiungeva:

fu merito dell'autorità comunale, del comitato promotore e di molti egregi cittadini che la commemorazione rivestisse tale carattere, tenendosi lontana da ogni manifestazione inopportuna di anticlericalismo o di violazione alle idee e alla libertà di tutti ${ }^{61}$.

Come ci aspetteremmo, i gruppi repubblicani locali, astenutisi dall'adesione alle manifestazioni giubilari "istituzionali" in ragione del marcato orientamento filo-sabaudo di esse, celebrarono tuttavia con particolare solennità gli anniversari legati alle figure di Pisacane, Mazzini, Garibaldi.

Sebbene, come abbiamo accennato in precedenza, nei decenni precedenti istituzioni ed élites governative avessero profuso sforzi per la «creazione di un culto di eroi nazionali dall'impronta conciliatrice» ${ }^{62}$, nel quale Garibaldi, Vittorio Emanuele II, Mazzini e Cavour divenivano strumenti di un unico disegno provvidenziale, la monumentalistica pubblica e le commemorazioni civiche continuavano a riflettere la contrapposizione tra la lettura filo-sabauda del processo di

\footnotetext{
${ }^{59}$ S.a., «Sorano, 21 settembre», in L'Ombrone, 1 ottobre 1911.

${ }^{60}$ Ibidem.

${ }^{61}$ Ibidem.

${ }^{62}$ RIALL, Lucy, Garibaldi: L'invenzione di un eroe, Roma-Bari, Laterza, 2007, p. XIII.
} 
unificazione e la concezione democratico-repubblicana del Risorgimento come movimento di popolo "tradito" dai governi liberali succedutisi al potere.

I monumenti dedicati a Vittorio Emanuele II e a Garibaldi condividevano dunque il posto d'onore nelle piazze e nei luoghi centrali di ogni città d'Italia ${ }^{63}$, mentre le celebrazioni risorgimentali erano organizzate sulla base di due calendari solo in parte sovrapponibili, quello istituzionale e quello elaborato dai gruppi democratici e repubblicani.

La memoria pubblica del Risorgimento fu condizionata, inoltre, dall'affacciarsi sull'arena politica d'epoca giolittiana dei cosiddetti "nuovi repubblicani”, per i quali occorreva sostanziare la visione mistica della patria di matrice mazziniana con la "promozione di una idea di democrazia insieme politica e sociale capace di valorizzare le libertà democratiche attraverso un'organizzazione decentrata e autonomistica dello Stato» ${ }^{64}$.

Nel corso del 1911 questo nuovo approccio condizionò il dibattito sulle misure speciali da adottare per lo sviluppo della Maremma e inflù̀ sulle modalità con le quali i gruppi repubblicani locali resero onore alle ricorrenze risorgimentali, come documentato sulle pagine di «Etruria nuova».

Le espressioni più frequenti del culto risorgimentale da parte dei repubblicani consistettero nell'apposizione di targhe e nella realizzazione di busti commemorativi ${ }^{65}$, spesso associate a conferenze e lezioni pubbliche.

La prima ricorrenza in ordine di tempo nel calendario "alternativo" adottato dai repubblicani era rappresentata dall'anniversario della proclamazione della Repubblica romana (9 febbraio) ${ }^{66}$, che in Maremma fu celebrato da un editoriale pubblicato su «Etruria nuova» e tramite l'adesione ad una vasta manifestazione di carattere regionale, promossa dalla federazione toscana del partito repubblicano.

Durante l'estate, inoltre, la frazione massetana di Tatti rese omaggio a Carlo Pisacane, ricordato da un'orazione pubblica il 13 agosto e, poche settimane dopo, da una lapida commemorativa nella quale la sua figura era associata a quella di Garibaldi, apposta su iniziativa dell'amministrazione municipale, di orientamento repubblicano ${ }^{67}$.

${ }^{63}$ ISNENGHI, Mario, Garibaldi fu ferito. Il mito, le favole, Roma, Donzelli Editore, 2010.

${ }^{64}$ RIDOLFI, Maurizio, «La Repubblica dell'avvenire ! Progetti e orizzonti repubblicani in Italia dal Risorgimento al tramonto della Monarchia», in Laboratoire italien, 19, 2017, URL:

< http://journals.openedition.org/laboratoireitalien/1258 > [consultato il 25 luglio 2018].

${ }^{65}$ Sulla "statuomanie" come fenomeno, nella sua prima fase rivoluzionario, tipico del lungo Ottocento si rimanda al testo di Maurice Agulhon, divenuto ormai classico, «La "statuomanie" et l'histoire», in Ethnologie française, 8, 2-3/1978, pp. 145-172.

${ }^{66}$ SEVERINI, Marco, «La Repubblica romana del 1849: una pagina di storia attuale», in Storia futuro. Rivista di storia e storiografia online, 26/2011, URL: < http://storiaefuturo.eu/repubblica-romana-1849-pagina-storiaattuale/ > [consultato il 25 luglio 2018].

${ }^{67}$ Archivio di Stato di Grosseto, Fondo Archivio Storico Post-Unitario del Comune di Grosseto, Serie 6 Carteggio Generale degli Affari, Faldone 20 Lutti, cittadinanze onorarie, onoranze, uomini illustri, mostre, monumenti e lapidi, Cartellina Ricordi a Garibaldi e Pisacane a Tatti, Estratto della delibera del Consiglio Comunale del 25 settembre 1911, oggetto: Ricordi a Garibaldi e Pisacane inaugurati a Tatti. In occasione 
Nel clima di fervente partecipazione creatosi nel 1911 in Maremma la memoria del Risorgimento, declinata in base alle diverse sensibilità politiche, era chiamata in causa anche in occasione di eventi eminentemente locali, coerentemente con quanto osservato da Valeria Galimi riguardo la monumentalistica realizzata nel grossetano in epoca liberale, nella quale «sebbene siano presenti alcuni monumenti relativi alla memoria nazionale, è soprattutto la costruzione del mito della Maremma [...] che sembra essere prevalente, o almeno, una forte connotazione locale» ${ }^{68}$.

In questo quadro, l'inaugurazione del monumento commemorativo ad Ettore Socci presso il lungomare di Follonica fu forse l'evento più partecipato nel calendario di manifestazioni pubbliche organizzate dai repubblicani in Maremma ${ }^{69}$.

Il 19 febbraio un busto in ghisa, copia del monumento collocato a Grosseto 4 anni prima, veniva inaugurato predisponendo una cerimonia corale che vide l'alternarsi di diversi oratori alla presenza di un folto pubblico, che comprendeva delegazioni provenienti da tutta la Toscana ed esponenti di numerose associazioni ${ }^{70}$.

Nel corso della manifestazione Socci fu più volte ritratto come l'erede della tradizione democratica risorgimentale, in virtù del suo impegno per uno sviluppo della Maremma improntato ai principi del repubblicanesimo e coerente con le «tradizioni di patriottismo e di abnegazione onde si onora la Maremma che da Cala Martina a Talamone ha tutta una storia di generosi ardimenti, che facilitarono l'unità italiana» ${ }^{71}$.

\section{Una «terra selvaggia e bella»: polemiche attorno ad una guida di viaggio}

Come accennato nel precedente paragrafo, le celebrazioni giubilari ebbero anche l'obbiettivo di riscattare l'immagine negativa che la letteratura di viaggio e gli osservatori stranieri avevano associato all'Italia, ed in particolare alle aree, come la Maremma, affette da condizioni ambientali e sociali "difficili"72.

dell'apposizione della lapide venne ristampato presso un editore locale il testamento politico di Pisacane. S.a., Testamento politico, pubblicato in occasione di un ricordo marmoreo in Tatti. In memoria di Carlo Pisacane, Grosseto, Tip. Perozzo, 1911; ROSSELLI, Nello, Carlo Pisacane nel Risorgimento italiano, Torino, Einaudi, 1977.

${ }^{68}$ GALIMI, Valeria, Memorie di pietra: monumenti a Siena e Grosseto fra Risorgimento e Grande Guerra, in BALLINI, Pier Luigi, COPPINI, Romano Paolo ( a cura di), Luoghi e simboli della memoria: le piazze della Toscana, Firenze, Regione Toscana, 2015, pp. 267-287, p. 284.

${ }^{69}$ Il fautore dell'iniziativa era stato Niccola Gierrazzi, reduce garibaldino e figura di riferimento per i gruppi repubblicani locali.

${ }^{70}$ LOTTI, A., «La grandiosa manifestazione di Follonica in onore di Ettore Socci», in Etruria Nuova, 26 febbraio 1911.

${ }^{71}$ Ibidem. Queste parole erano attribuite a Gaetano Badii, storico massetano figlio di un volontario garibaldino, noto per aver scritto l'opera Massa Marittima: la Brescia Maremmana nella storia del risorgimento italiano e l'opera del dott. Apollonio Apolloni ufficiale garibaldino, Milano, L. Trevisini, 1912.

${ }^{72} \mathrm{Si}$ veda, in particolare, il citato studio di Baioni riguardo il Ravennate e la Romagna: BAIONI, Massimo, Rituali in provincia, cit. 
Anche nel grossetano, peraltro, il tentativo di rilanciare il territorio maremmano si scontrava con gli stereotipi che impedivano di apprezzare i pur limitati progressi compiuti da quest'area nel corso del XIX secolo, scatenando la vis polemica della popolazione sui periodici locali, come avvenne anche nel 1911 in occasione della pubblicazione di una guida illustrata de Il litorale Maremmano curata da Carlo Alberto Nicolosi ${ }^{73}$.

La raffigurazione della Maremma di Nicolosi era infatti dominata dalla presenza incombente della malaria, minaccia costante per la modernizzazione della regione e ostacolo ad una più ampia conoscenza di essa:

(il lettore medio) tutto ignora di quest'ampia regione tra i monti ed il mare, magnifico deserto di boschi e di verdura, la cui fecondità si consuma espandendosi liberamente in una vegetazione folle e lussureggiante; tutto ignora di questa terra selvaggia e bella, paradiso di cacciatori e di briganti, ricchezza ai proprietari lontani, inferno e miseria ai suoi abitatori, tramanti per la terzana. La ignora nella sua storia più che millenaria, nei rari paesi dove si nascondono umili e squisite opere d'arte, nella feracità meravigliosa del suolo, proverbiale dagli antichissimi tempi, nella varia e mutevole bellezza del paesaggio, diverso da ogni altro ${ }^{74}$.

Nella guida di Nicolosi, dunque, la Maremma si ammantava di un fascino oscuro, preservato dallo stesso ritardo di sviluppo che affliggeva le comunità che popolavano

questo paese così strano, ridente a volte nel suo aspetto selvaggio, a cui non siamo più abituati, più spesso di una tragica tristezza, sempre però ampio e severo, di una grande e suggestiva bellezza, che non a tutti si impone di primo acchito, ma che per essere compresa ha bisogno di riflessione e di convincimento ${ }^{75}$.

Nelle righe successive Nicolosi insisteva quasi morbosamente sullo stereotipo della Maremma maledetta, riportando alcuni proverbi e detti locali improntati al pessimismo più cupo, pur riconoscendo alcuni recenti segnali di crescita nel miglioramento dei collegamenti ferroviari, nella presenza di prospere fattorie, nell'affacciarsi di una «generazione nuova» di giovani non più segnati come i padri dai segni evidenti delle febbri ma sani e vigorosi:

Da Cecina a Follonica ormai la regione, per fortuna sua, non ha più il diritto di chiamarsi Maremma nel senso tradizionale che suol darsi a questo nome. Coltivata e popolosa, risorta al lavoro, essa non può più pretendere che al titolo d'anticamera della maremma, ed è quasi una delusione per chi [...] invece del morto paese che credeva incontrare, scorge questa pianura

\footnotetext{
${ }^{73}$ NICOLOSI, Carlo Alberto, Il litorale Maremmano. Grosseto-Orbetello, Bergamo, Istituto italiano di arti grafiche, 1910.

${ }^{74}$ Ibidem, p. 13.

${ }^{75} \mathrm{Ibidem}$, p. 17.
} 
verde, interrotta qua e là da appezzamenti di terra rosso bruna, ben pettinata a e frequente di abitati $^{76}$.

Tuttavia, per Nicolosi la Maremma autentica era incarnata da «la piccola stazione di Follonica, bassa lurida e fuligginosa, sgangherata dal lungo abbandono, è ben la stazione più maremmana che si possa immaginare» o dalla piana di Grosseto, «vasta e immobile sotto una pesantezza d'aria afosa, intensamente verde o squallidamente gialla secondo le stagioni, silenziosa come una tomba» ${ }^{77}$, che dalla presenza degli Etruschi aveva tratto

l'essenza del loro carattere pessimista e dubbioso, col quale la nostra modernità psicologica ha tanti punti di contatto. Qui, nella desolazione delle terre incolte da dove il benessere è scomparso con loro, qui solo intendiamo la sconsolata tristezza della loro religione, a cui nessuna speranza arrideva $^{78}$

L'opera di Nicolosi suscitò aspre critiche nell'opinione pubblica maremmana, al punto che dalle pagine de «L'Ombrone» l'autore fu accusato di essersi «preoccupato assai più della frase immaginosa, anziché della riproduzione esatta delle sue condizioni di un paese e del suo stato di vita», conformandosi a topoi letterari che sarebbe stato meglio abbandonare "per amor di patria, prima che per il loro gusto antiquato» ${ }^{79}$.

Nicolosi chiese ed ottenne di pubblicare una replica alle accuse mossegli sulle pagine dell'Ombrone, nella quale egli negava ogni intento denigratorio, rimarcando al contrario la sua «adorazione» per la Maremma, «terra selvaggia e bella finora troppo dimenticata», e rilevava un certo accanimento nei confronti della sua opera, in parte derivante da un interpretazione troppo letterale delle espressioni poetiche utilizzate per descrivere il litorale maremmano ${ }^{80}$.

In seguito alla replica dell'autore, la redazione dell'Ombrone decise di porre fine alla polemica, esprimendo apprezzamento per la "palinodia" di Nicolosi e, tuttavia, lasciando l'ultima parola sulla questione ad un anonimo lettore, che avrebbe espresso in una lettera al periodico il suo rammarico per il mancato riconoscimento nelle pagine de Il litorale Maremmano del «nuovo fermento di attività che si verifica in ogni campo in Maremma per merito principale, anzi esclusivo, dei maremmani» ${ }^{81}$.

\footnotetext{
${ }^{76}$ Ibidem, pp. 21-22.

${ }^{77}$ Ibidem, p. 24.

${ }^{78}$ Ibidem, p. 25.

${ }^{79}$ R.O., "A proposito di un libro», in L'Ombrone, 12 febbraio 1911.

${ }^{80}$ Nicolosi si dichiarò particolarmente stupito per l'indignazione suscitata da una frase nella quale definiva la Maremma dei tempi passati un "paradiso dei briganti»: «ma potevo io mai supporre che la si potesse prendere alla lettera, proprio oggi che al brigantaggio in Italia non credono più nemmeno gli albergatori svizzeri?» NICOLOSI, Carlo Alberto, «Ancora sulla monografia della nostra Maremma», in L'Ombrone, 26 febbraio 1911.

${ }^{81}$ S.a., «Sempre il "litorale maremmano"», in L'Ombrone, 5 marzo 1911.
} 
La polemica non si rivelò uno sterile duello verbale, ma, al contrario, apportò ulteriore linfa ad una riflessione pubblica sul passato, sul presente e, soprattutto, sul futuro della Maremma che tenne banco in Parlamento, nelle istituzioni periferiche e sulle pagine delle testate locali.

\section{Il "Risorgimento mancato" della Maremma grossetana}

Come anticipato, prendendo spunto da un discorso tenuto da Pio Viazzi in Parlamento nel dicembre precedente, nei primi mesi del 1911 il dibattito sull'isolamento e il ritardo di sviluppo in Maremma acquisì nuovo vigore, mescolando riferimenti al quadro politico nazionale con polemiche di carattere localistico.

La prima testimonianza degna di interesse è, dunque, offerta dal testo illustrato da Viazzi in Parlamento, riportato da «Etruria Nuova», nel quale egli puntualizzava:

se ora la Maremma toscana ha creduto bene di affermare i propri diritti è perché in verità non solo crede sia un atto di perfetta giustizia, ma di puro dovere, il suo intervento tra le altre regioni d'Italia per proclamare le sue oneste aspirazioni al proprio risorgimento, dacché essa vuole affermarsi una buona volta nella generale vita economica del paese.

Viazzi proseguiva in un riferimento alle «ricchezze della Maremma», notevoli nonostante gli elementi di crisi che affliggevano l'economia della regione:

Tutto il territorio ha una varietà ammirabile di coltivazione, dalla flora tropicale del giardino di acclimatazione di Port'Ercole, dagli aranci e limoni del Ricasoli a Port'Ercole, di Santo Stefano, di Scarlino, alla zona intermedia degli ulivi, dei lecci, delle sughere, fino ai faggeti della montagna. Il paesaggio è vario, ora fiero, ora ridente. [...] Le varie civiltà italiche sono pronte ad offrire quivi agli amatori tutti i loro documenti [...] Solo perché io sono ben certo del risorgimento di questa regione, me ne faccio caldo patrocinatore ${ }^{82}$.

L'orazione di Viazzi si concludeva con un cenno all'opera meritoria dei Lorena nel campo delle bonifiche, puntualizzando però che «i maremmani non vogliono ritornare indietro nella storia: essi pensano all'avvenire» ${ }^{83}$.

Come anticipato, la proposta di estensione dei benefici per la città di Napoli anche al territorio maremmano, avanzata da Viazzi, non venne accolta ${ }^{84}$ : in un'intervista resa al «Nuovo Giornale» e riportata da «Etruria Nuova», il parlamentare rintuzzava le polemiche scatenate dall'insuccesso della sua iniziativa, stigmatizzando l'ostilità di alcune regioni (Piemonte e Liguria in primis) che

\footnotetext{
${ }^{82}$ S.a., «Il Problema della Maremma dinanzi al Parlamento», in Etruria Nuova, 8 gennaio 1911.

${ }^{83}$ Ibidem.

${ }^{84}$ La Maremma poté invece godere dei benefici attribuiti all'Agro Romano, ma solamente a partire dal 1919.
} 
aveva indotto il governo a negare le provvidenze richieste per timore della "concorrenza" recata da una Maremma risanata, e, più in generale, per un miope campanilismo.

L'intervista offriva al deputato una nuova occasione per sintetizzare le priorità maremmane nella ripresa delle bonifiche, nella colonizzazione delle aree meno densamente popolate e nel miglioramento della rete stradale; si sottolineava nel contempo l'urgenza di interventi a favore dei piccoli porti (questione strettamente intrecciata al completamento della rete ferroviaria con il tronco Santo Stefano-Orbetello e con il collegamento tra Orbetello e Orvieto) e delle scuole locali (si ricordava che la provincia di Grosseto non accoglieva né un liceo né un istituto tecnico) ${ }^{85}$.

Scartata l'ipotesi di estendere alla Maremma toscana i benefici previsti per altre aree svantaggiate, il Governo, complice il bisogno di consenso in previsione della guerra di Libia, ritenne di dover fornire una risposta concreta all'emergenza occupazionale ed ambientale della Maremma, non fronteggiabile tramite le sole risorse degli enti locali.

Nel 1911 venne dunque costituita su iniziativa governativa una Commissione speciale pel risorgimento economico della Maremma, incaricata di studiare le problematiche dell'area e redigere un piano d'azione.

I lavori della Commissione si conclusero con una visita ufficiale da parte del ministro dei Lavori pubblici Ettore Sacchi, il quale tenne a Grosseto un discorso pubblico denso di elementi di interesse.

Il sindaco di Grosseto, introducendo l'intervento di Sacchi, aveva sottolineato come i benefici recati dagli interventi attuati in epoca granducale sul patrimonio infrastrutturale ed il tessuto socio-economico maremmano fossero stati vanificati nel corso dei primi decenni postunitari, danneggiando gravemente un'area che non reclamava altro «se non il modesto desiderio di essere visitata, di essere conosciuta, di essere compresa ed incoraggiata».

Il Ministro Sacchi prese spunto da queste affermazioni per ribadire l'impegno del governo a favore della Maremma, all'interno del più ampio obbiettivo dell'«unificazione economica» della Penisola.

Ridimensionando, prevedibilmente, i meriti dei Lorena, Sacchi giustificava parzialmente il ritardo di sviluppo della Maremma con la «naturale difficoltà $\mathrm{d}(\mathrm{e}) \mathrm{i}$ problemi» dai quali essa era interessata, e preannunciava un vasto programma di investimenti ${ }^{86}$.

Nonostante le rassicurazioni del ministro, nei mesi successivi i rappresentanti delle istituzioni locali si attivarono per richiamare il Governo al rispetto degli impegni assunti e, nel mese di

\footnotetext{
${ }^{85}$ FIORINI, M., «Il Rinascimento della Maremma Toscana», in Etruria Nuova, 15 gennaio 1911.

${ }^{86}$ I soli interventi infrastrutturali per bonifiche, sistemazioni di porti e coste e miglioramento della viabilità previsti per il successivo decennio avrebbero richiesto un finanziamento di circa 46 milioni di lire. R.O., «La nuova Italia è impegnata al risorgimento economico della Maremma Toscana. La visita del Ministro Sacchi a Grosseto», in L'Ombrone, 19 marzo 1911.
} 
luglio, alcuni membri del neo-costituito Comitato di agitazione pro-Maremma vennero ricevuti da Giolitti, tornato di recente a ricoprire la carica di primo ministro ${ }^{87}$.

In un editoriale di fine luglio, tuttavia, «L'Ombrone» esprimeva scetticismo riguardo l'affidabilità degli impegni assunti dal ministro Sacchi e, più in generale, rimarcava l'inutilità delle recenti inchieste conoscitive sulla "Cenerentola d'Italia» ${ }^{88}$. Persino il ministro all'agricoltura, industria e commercio Ministro Francesco Saverio Nitti, come riportavano alcune fonti, aveva posto in dubbio l'utilità delle provvidenze alla Maremma suggerendo «con sarcasmo» che sarebbe stato più conveniente corrispondere ad ogni maremmano la cifra necessaria per eguagliare il reddito medio individuale italiano piuttosto che realizzare i prospettati interventi sul territorio ${ }^{89}$.

L'autore dell'articolo ribadiva la contrarietà degli abitanti dell'area ad ogni forma di elemosina, e, nello stesso tempo, suggeriva l'adozione di forme di protesta eclatanti, tra le quali la presentazione simultanea delle dimissioni da parte di tutte le cariche pubbliche, contro le lungaggini e le ambiguità della burocrazia governativa, considerando che «la promessa di studiare ancora è erba trastulla e di questo non ci dobbiamo ne possiamo appagare». L'articolo era corroborato da una lettera del presidente della Deputazione provinciale di Grosseto, l'avvocato Armando Pastorelli, che rispondeva alle critiche mosse dal giornale il 2 luglio, circa la «deplorevole inerzia» dell'Ufficio tecnico provinciale, che, in realtà, aveva sospeso le ordinarie attività di manutenzione dopo che la Commissione pro-Maremma aveva prospettato la nazionalizzazione di alcune strade provinciali, senza ulteriori atti concreti da parte delle istituzioni centrali90.

$\mathrm{Fu}$, dunque, per scuotere l'inerzia che sembrava offrire i maggiori ostacoli alla rinascita della Maremma che il Comitato mandamentale di Campiglia Marittima raccolse una serie di segnalazioni e proposte all'interno di una relazione, data alle stampe anch'essa nel $1911^{91}$.

Gli estensori della relazione presentarono il proprio lavoro sottolineando che «il movimento iniziatosi nella Maremma per la sua rigenerazione economica, non è opera delle sole sfere Ufficiali, o di pochi, ma azione attiva di un popolo che al sentimento del dovere, non disgiunge quello della propria dignità, che sente anzi in massimo grado, e sa di possedere ricchezze, energie e forze poderose, solo compresse da cause certo non imputabili all'iniziativa privata» ${ }^{92}$.

Lo scritto enucleava le problematiche della Maremma grossetana, andando ben oltre i confini del mandamento di Campiglia e suggerendo le possibili direttrici dell'intervento statale,

\footnotetext{
${ }^{87}$ S.a., «Il Comitato Pro-Maremma ricevuto dall'on. Giolitti», in L'Ombrone, 16 luglio 1911. La delegazione comprendeva i parlamentari eletti nella Maremma toscana e pisana (Piero Ginori Conti, Gaspero Ciacci e Pio Viazzi), i sindaci di alcuni comuni (spicca l'assenza del sindaco di Grosseto), rappresentanti delle deputazioni provinciali di Pisa e Grosseto, della Camera di Commercio di Grosseto e di altre istituzioni.

${ }^{88}$ S.a., «La vana promessa», in L'Ombrone, 23 luglio 1911.

${ }^{89}$ Ibidem.

${ }^{90}$ Ibidem.

${ }^{91}$ S.a., Per il Risorgimento economico della Maremma. Relazione del Comitato Mandamentale di Campiglia Marittima, Livorno, Tip. Belforte, 1911.

${ }^{92}$ Ibidem, p. 7.
} 
rimproverando ai governi liberali succedutisi dopo l'unificazione «l'inerzia» e l'incapacità di raccogliere l'eredità Granducale completando le bonifiche e valorizzando l'area ${ }^{93}$.

Il disinteresse delle classi dirigenti del centro era dunque la causa del persistere di problemi di lunga data, come «la malaria, lo spopolamento, l'incoltura delle nostre terre, il difetto di industrie, l'improduttività delle miniere», ai quali si andavano sommando criticità più recenti, tra le quali l'incremento del costo del lavoro ${ }^{94}$.

Largo spazio era riservato alla questione delle bonifiche, con l'auspicio di eliminare le pastoie burocratiche che ostacolavano il completamento delle opere idrauliche con una tempistica che, in condizioni propizie, sarebbe stata di circa un decennio, ricorrendo eventualmente alla creazione di un ente autonomo nel quale fosse rappresentata anche la provincia di Grosseto ${ }^{95}$.

Altrettanto importanti apparivano le piccole opere di bonifica necessarie per completare la sistemazione idraulica della zona, interessata da difficoltà nell'approvvigionamento e distribuzione di acqua potabile e nella manutenzione di scarichi e canali ${ }^{96}$.

Le risorse dei comuni, ma anche quelle dei proprietari privati, erano ritenute insufficienti per finanziare un così ampio piano di interventi, pertanto si auspicava l'approvazione di agevolazioni creditizie e fiscali, coinvolgendo Consorzi e istituzioni agrarie cooperative ${ }^{97}$; nel contempo si individuava nell'istituzione di nuove cattedre ambulanti di agricoltura un'ulteriore passaggio chiave per lo sviluppo rurale ${ }^{98}$.

Si suggeriva, inoltre, l'abrogazione dei vincoli demaniali gravanti su vaste aree e il loro affidamento a privati, pur tutelando i diritti delle comunità locali ${ }^{99}$ mentre, anticipando una visione dell'economia rurale che si sarebbe affermata solo nel tardo Novecento, si incoraggiava l'integrazione tra agricoltura e industrie di lavorazione dei prodotti, poiché «la Maremma per la molteplicità e varietà dei suoi prodotti, e per la naturale freschezza dei terreni suscettibili ad ogni coltivazione di carattere industriale, offre e potrebbe ancor più offrire ricchezze inesauribili di materie prime alle industrie, né è completamente priva di forze motrici naturali», nonostante «l'opera distruggitrice» esercitata da una eccessiva fiscalità ${ }^{100}$. Secondo gli autori, le criticità del settore minerario erano imputabili alle deficienze nel trasporto ferroviario, all'aumento del costo del lavoro e al peso degli oneri imposti dalle riforme per la tutela dei lavoratori di recente introduzione, con l'effetto di incrementare l'emigrazione e indebolire le aziende nazionali a vantaggio di quelle straniere ${ }^{101}$.

\footnotetext{
${ }^{93}$ Ibidem, p. 8.

${ }^{94}$ Ibidem.

${ }^{95}$ Ibidem, p. 10.

${ }^{96}$ Ibidem, p. 11.

${ }^{97}$ Ibidem, pp. 13-14.

${ }^{98}$ Ibidem, pp. $14-15$.

${ }^{99}$ Ibidem, p. 16.

${ }^{100}$ Ibidem, p. 17.

${ }^{101}$ Ibidem, pp. 17-19.
} 
Lo spopolamento generato dal declino del tessuto economico locale avrebbe a sua volta privato la Maremma delle risorse umane indispensabili per colonizzare e valorizzare le aree sottratte alle paludi, «giacché, è inutile negarcelo, trascorreranno molti anni prima che il forestiere, venga a Noi fiducioso, e vi porti affetti ed energie pari a quelle di cui è capace ogni figlio per il Paese natio» ${ }^{102}$.

Un capitolo a parte era dedicato alle cattive condizioni dell'istruzione pubblica locale, causate dalla mancanza di scuole superiori ad indirizzo tecnico che formassero i lavoratori dei diversi settori e dalla scarsità di risorse comunali disponibili per interventi igienico-strutturali e gestionali.

Nella sezione finale, inoltre, gli autori mettevano in guardia dal rischio che la cosiddetta Maremma pisana, affetta da criticità ancora più gravi rispetto al territorio grossetano, potesse monopolizzare le risorse statali una volta approvati interventi a favore della maremma toscana nel suo insieme ${ }^{103}$.

La conclusione offriva un vivido ritratto delle comunità maremmane, piegate, ma non sconfitte da problemi secolari e dai drammi della modernità: «la Maremma non chiede privilegi, ma rivendica diritti; ed aspira all'ascensione che le meritano la virtù dei suoi Cittadini laboriosi, la feracità del suo suolo, le ricchezze di cui le fu prodiga natura» ${ }^{104}$.

Nell'anno del cinquantenario dall'unificazione, la relazione non poteva non concludersi con un richiamo al legame tra patriottismo e sviluppo, auspicando che il «diritto» delle genti maremmane potesse «trionfare in piena armonia agli interessi della nazione» ${ }^{105}$.

\section{Conclusioni}

Le testimonianze analizzate all'interno di questo contributo confermano come le celebrazioni giubilari siano state contrassegnate dall'intimo legame, e l'apparente contraddizione, tra la celebrazione dell'epopea risorgimentale, in una visione progressiva dei primi cinquant'anni di esistenza del Regno d'Italia, e la riflessione sulle criticità del periodo postunitario e i perduranti squilibri socio-economici tra le diverse aree della Penisola.

L'elaborazione di una liturgia che declinasse e reinterpretasse localmente i motivi presenti nelle celebrazioni giubilari a livello nazionale, pose inevitabilmente in luce le contrapposizioni ideologiche che dividevano, in primo luogo, monarchici e repubblicani, attribuendo significati ed esiti diversi all'epopea risorgimentale.

${ }^{102}$ Ibidem, p. 20. Tra le proposte avanzate nella relazione figura anche l'impianto di una linea telefonica, ritenuta cruciale in ragione della relativa distanza tra i diversi comuni del mandamento.

${ }^{103}$ Ibidem, p. 26.

${ }^{104}$ Ibidem, p. 27.

${ }^{105}$ Ibidem, p. 28. 
Nel contempo, le celebrazioni giubilari facilitarono il consolidamento dell'identità locale, nel più ampio contesto di quel processo di trasmissione di forme celebrative e riferimenti culturali dal centro alla periferia efficacemente evidenziato da Roberto Balzani con riferimento alla Romagna ${ }^{106}$.

Similmente a quanto verificatosi a livello nazionale e in altre regioni, dunque, la ricorrenza del cinquantenario stimolò la riflessione retrospettiva sullo sviluppo, attuato o mancato, delle comunità locali, che nella Maremma grossetana si colorò di toni particolarmente polemici.

L"'assenza" dei governi liberali, poco o nulla interessati alla risoluzione dei problemi ambientali, sociali ed economici dell'area, fu stigmatizzata non solo sulle pagine di «Etruria Nuova», locale periodico repubblicano, ma anche su quelle de «L'Ombrone», voce dei liberalimoderati.

Nel contempo, si accese la polemica nei confronti di una letteratura di viaggio che proponeva lo stereotipo dark di una Maremma malsana e condannata al sottosviluppo, riecheggiando, anche in questo caso, analoghe rivendicazioni contro i pregiudizi negativi degli osservatori stranieri avanzate in occasione delle celebrazioni giubilari a livello nazionale.

In questo quadro, pertanto, attaccamento alla comunità locale e amor di patria si sostanziavano a vicenda nella considerazione del contributo che una Maremma risanata e sviluppata avrebbe recato al benessere della nazione, pur non rinunciando al ricordo nostalgico dell'epoca d'oro granducale.

L'intreccio tra celebrazione e rivendicazione verificatosi nella Maremma grossetana nel 1911, dunque, conferma la contraddizione insita nelle manifestazioni giubilari, a sua volta espressione della frammentarietà e dell'incompiutezza del processo di unificazione.

Da un punto di vista metodologico, i materiali proposti suggeriscono l'utilità di un nuovo approccio ai contenuti culturali della liturgia politica diffusa in epoca liberale, che non si riduca ad una puntuale esegesi delle fonti a disposizione, priva di un autentico valore euristico, e si interroghi, piuttosto, sui significati di feste e celebrazioni per illuminare aspetti diversificati della storia sociale, economica, ambientale.

L'ampio ventaglio di categorie interpretative applicate allo studio della memoria pubblica e delle festività civili negli ultimi anni sembra indicare che il rinnovamento di questo filone di studi è già in atto e che le celebrazioni nazionali costituiscono oramai uno dei terreni privilegiati per l'analisi delle grandi trasformazioni della società contemporanea.

${ }^{106}$ BALZANI, Roberto, La Romagna. Storia di un'identità, Bologna, Il Mulino, 2012. 


\section{L'AUTORE}

Elisa TIZZONI, (La Spezia, 1983), $\mathrm{PhD}$ in Storia contemporanea, ha svolto incarichi di ricerca ed insegnamento presso le Università di Firenze, Pisa, Nizza, Salisburgo e presso altre istituzioni culturali; nel 2017 ha ottenuto una Postgraduate Vibeke Sørensen Grant presso l'Historical Archives of the European Union a Firenze. Attualmente è visiting Fellow presso il Department of History and Civilization dell'Istituto Universitario Europeo di Firenze e Cultore della Materia presso il Dipartimento di Storia e Civiltà dell'Università di Pisa.

URL: < http://www.studistorici.com/progett/autori/\#Tizzoni > 